\title{
Strong partitioning of soil bacterial community composition and co-occurrence networks along a small-scale elevational gradient on Zijin Mountain
}

\author{
Xu Liu ${ }^{1,2}$, Teng Yang ${ }^{1,2}, \mathrm{Yu} \mathrm{Shi}^{3}$, Yichen Zhu ${ }^{4}$, Mulin $\mathrm{He}^{4}$, Yunke Zhao ${ }^{4}$, Jonathan M. Adams ${ }^{5}$, Haiyan Chu ${ }^{1,2,{ }^{*}}$ \\ 1 State Key Laboratory of Soil and Sustainable Agriculture, Institute of Soil Science, Chinese Academy of Sciences, Nanjing 210008, China \\ 2 University of Chinese Academy of Sciences, Beijing 100049, China \\ 3 State Key Laboratory of Crop Stress Adaptation and Improvement, School of Life Sciences, Henan University, Kaifeng 475004, China \\ 4 High School Affiliated to Nanjing Normal University, Nanjing 210003, China \\ 5 School of Geographic and Oceanographic Sciences, Nanjing University, Nanjing 210006, China
}

\section{H I G H L I G H T S}

- Soil bacterial community composition strongly differed along a short elevational gradient.

- Soil $\mathrm{pH}$ and elevation were significantly correlated with soil bacterial community composition.

- Degree scores, betweenness centralities, and composition of network hubs differed among elevations.

\section{ARTICLE INFO}

Article history:

Received June 30, 2021

Revised September 14, 2021

Accepted September 22, 2021

\section{Keywords:}

Elevational distribution

Soil pH

Bacterial community composition

Co-occurrence network

\section{GRAPHICAL ABSTRACT}

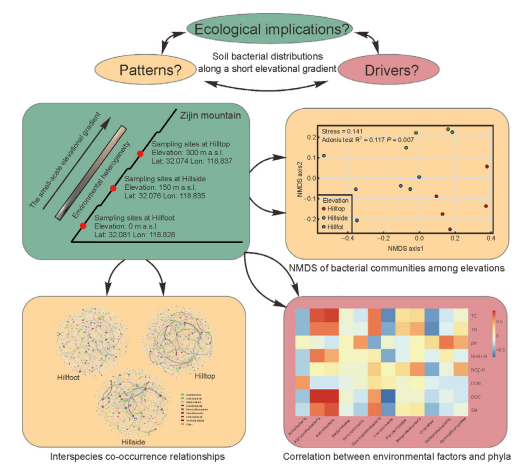

A B S T R A C T

The elevational distributions of bacterial communities in natural mountain forests, especially along large elevational gradients, have been studied for many years. However, the distributional patterns that underlie variations in soil bacterial communities along small-scale elevational gradients in urban ecosystems are not yet well understood. Using Illumina MiSeq DNA sequencing, we surveyed soil bacterial communities at three elevations on Zijin Mountain in Nanjing City: the hilltop (300 m a.s.l.), the hillside (150 $\mathrm{m}$ a.s.l.), and the foot of the hill $(0 \mathrm{~m}$ a.s.I.). The results showed that edaphic properties differed significantly with elevation. Bacterial community composition, rather than alpha diversity, strongly differed among the three elevations (Adonis: $\mathrm{R}^{2}=0.12, P<0.01$ ). Adonis and DistLM analyses demonstrated that bacterial community composition was highly correlated with soil $\mathrm{pH}$, elevation, total nitrogen (TN), and dissolved organic carbon (DOC). The degree scores, betweenness centralities, and composition of keystone species were distinct among the elevations. These results demonstrate strong elevational partitioning in the distributions of soil bacterial communities along the gradient on Zijin Mountain. Soil pH and elevation together drove the smallscale elevational distribution of soil bacterial communities. This study broadens our understanding of distribution patterns and biotic co-occurrence associations of soil bacterial communities from large elevational gradients to short elevational gradients.

(C) Higher Education Press 2021

\footnotetext{
* Corresponding author

E-mail address: hychu@issas.ac.cn (H. Chu)
} 


\section{Introduction}

The study of microbial distribution patterns is one of the major focuses of microbial ecology. Such studies have generated essential insights for the development of classic theories of biodiversity and have provided new perspectives on the response of microbial communities to global change (Ladau et al., 2018; Chu et al., 2020). Although the elevational distributions of terrestrial animals and plants have been studied for many years, studies of the elevational patterns in microbial diversity only gained popularity after the beginning of the 21st century. Most previous studies on elevational patterns of microbial diversity have been carried out in natural ecosystems and along large-scale elevational gradients (Fierer et al., 2011; Shen et al., 2013; Singh et al., 2014; $\mathrm{Li}$ et al., 2020). Compared with small-scale elevational gradients, large-scale elevational gradients have larger elevational intervals and absolute elevations, with large hydrothermal gradients and highly heterogeneous soil environments. Different factors have been found to affect microbial distribution along elevational gradients. For example, soil $\mathrm{pH}$ was the optimal predictor of soil bacterial distribution along an increasing elevational gradient from 530 to $2200 \mathrm{~m}$ a.s.l. on Changbai Mountain (Shen et al., 2013). The aboveground plant community was regarded as the crucial factor that drove the distribution of diazotrophic bacteria along an elevational gradient from 1800 to $4100 \mathrm{~m}$ a.s.l. on Gongga Mountain (Wang et al., 2019). Along small-scale elevational gradients, soil microbial communities may be less affected by dispersal limitation and environmental filtering (due to the relatively small environmental variance). Soil microbial distribution patterns along smaller elevational gradients, the mechanisms that shape them, and the ways in which they differ from those along larger gradients are poorly understood.

Characterizing the ecological implications and functions of soil microbes, as well as their interactions with edaphic properties and their contributions to complex ecological processes, provides crucial insights into soil ecological functions (Röttjers and Faust, 2018). Network analyses, originally developed to study social relationships and the internet, can also be used to unravel complex relationships among microbial taxa (Toju et al., 2018). By adopting a network analysis framework, we can gain new insight into microbial elevational distributions and reveal previously unnoticed microbial co-occurrence patterns, including strong non-random interactions, niche specialization, vital ecological processes, and their implications (Faust and Raes, 2012; Zhang et al., 2014). The topology of microbial co-occurrence networks can depict the characteristics of co-occurrence patterns at multiple functional and taxonomic levels (Chaffron et al., 2010). For example, Ma et al. (2016) investigated the bacterial, fungal, and archaeal interaction relationships in forest ecosystems of eastern China and found that soil microbial communities in northern forests had closer interactions (i.e., degree scores) and lower interaction influence (i.e., betweenness centrality) than those in southern forests. Moreover, such topology-based information can be used to identify network hubs (also called keystone species in microbial ecology research). Instead of searching for the species that cause community variations, this method can accurately identify species that play important roles in microbial interactions. For example, Fan et al. (2019) used co-occurrence network analysis to reveal that Geobacter spp. was regarded as a keystone species linked with functionality losses under long-term fertilization conditions. Considerable efforts have been devoted to the characterization of microbial diversity and community composition along elevational gradients; however, only a few studies have focused on microbial interactions along elevational gradients (Farrer et al., 2019). Combined with studies of microbial community structure, research on microbial interactions will provide new information and perspectives on patterns of soil microbes.

Urban park soil plays an important role in urban ecosystems and has thus attracted increasing attention in recent years (Li et al., 2018; Yang et al., 2021), particularly in locations such as New York City's Central Park (Ramirez et al., 2014) and Beijing's Tiantan Park (Xu et al., 2014). These ecosystems may have characteristics distinct from those of natural ecosystems, reflecting the effects of human activity and regional climate. For example, the heat-island effect of the urban microclimate leads to temperature fluctuations, and human activities have an unquantifiable impact on microbial ecological processes (Bai et al., 2017). Recently, biogeographic distributions of soil microbiomes in urban landscape ecosystems have been reported (Xu et al., 2014; Huot et al., 2017; Yang et al., 2021). For example, Xu et al. (2014) revealed that Proteobacteria, Actinobacteria, Acidobacteria, and Planctomycetes were the dominant bacterial taxa in urban park soils in China, and multiple factors, including soil $\mathrm{pH}$, annual average precipitation, and annual average temperature, jointly drove the variation in bacterial community structure. In addition, Yang et al. (2021) surveyed bacterial communities in urban soil from 10 major cities in China and found strong distance-decay relationships and clear geographic zoning of the communities. However, the distribution patterns and driving mechanisms of soil microbes along urban elevational gradients are largely unknown. Our research region Zijin Mountain is an open mountain park and located in the urban area of Nanjing City, Eastern China. Large numbers of tourists and residents visit publicly accessible Zijin Mountain for leisure on a daily basis. Furthermore, Zijin Mountain is home to a wide diversity of plants (Dong et al., 2011) and soil microbes (Song et al., 2004; Qu et al., 2020). Therefore, Zijin Mountain provides an excellent platform for the study of bacterial distribution patterns along a small-scale elevational gradient in an urban landscape ecosystem. In this study, we asked two questions: 1) Do bacterial diversity and community composition differ along a small-scale elevational gradient, and if so, what forces drive these differences? 2) Do microbial co-occurrence 
network patterns, including network topologies and the composition of network hubs, differ among different elevations?

\section{Materials and methods}

\subsection{Site selection and soil sampling}

Soil samples were collected on Zijin Mountain $\left(32^{\circ} 5^{\prime} \mathrm{N}\right.$, $118^{\circ} 48^{\prime} \mathrm{E}$ ) in Nanjing City, Eastern China. Zijin Mountain has an area of 3008.8 ha and a peak height of $448.9 \mathrm{~m}$ a.s.l. (Fig. S1; Wang et al., 2020). The climate of Zijin Mountain has northern subtropical characteristics, with four distinct seasons throughout the year. The annual mean temperature is $15.4^{\circ} \mathrm{C}$, and the annual mean precipitation is $1013 \mathrm{~mm}$. The soils in Zijin Mountain are mainly composed of Albeluvisols (Soil order, WRB).

Soils were sampled from three elevations at $150 \mathrm{~m}$ elevational intervals on Zijin Mountain on March 14, 2016: the hilltop (300 $\mathrm{m}$ a.s.I.), the hillside (150 $\mathrm{m}$ a.s.I.), and the foot of the hill (hillfoot, $0 \mathrm{~m}$ a.s.I.). A conceptual map of the sampling scheme is shown in Fig. S1. At each elevation, we established five $10 \mathrm{~m} \times 10 \mathrm{~m}$ plots at least $30 \mathrm{~m}$ apart and collected at least 5 cores per plot at a depth of $0-10 \mathrm{~cm}$. These were subsequently combined and mixed in an aseptic bag to form one independent sample at the plot level (15 soil samples in total). Visible roots and residues were removed from the soil samples before homogenizing the soil at the plot level. The fresh soils were sieved (2-mm mesh) and divided into two subsamples for different uses. One subsample was stored at $4^{\circ} \mathrm{C}$ and used for measurement of soil chemical and physical properties. The other subsample was stored at $-20^{\circ} \mathrm{C}$ and used for DNA extraction.

\subsection{Soil chemical and physical analyses}

Eight edaphic variables were measured: soil moisture (SM), soil $\mathrm{pH}$, total $\mathrm{C}(\mathrm{TC})$, total $\mathrm{N}(\mathrm{TN})$, nitrate- $\mathrm{N}\left(\mathrm{NO}_{3}{ }^{-} \mathrm{N}\right)$, ammonium- $\mathrm{N}\left(\mathrm{NH}_{4}{ }^{+}-\mathrm{N}\right)$, dissolved organic nitrogen (DON), and dissolved organic carbon (DOC). SM was measured gravimetrically by drying $10 \mathrm{~g}$ of fresh soil until it reached a constant weight. Soil $\mathrm{pH}$ was measured with a $1: 5$ soil to boiled deionized water suspension using a Thermo Orion-868 $\mathrm{pH}$ meter (Thermo Fisher, Waltham, MA, USA). Soil TC and $\mathrm{TN}$ were determined using a $\mathrm{C}-\mathrm{H}-\mathrm{N}$ elemental analyzer (2400 II CHN; PerkinElmer, Boston, MA, USA). DOC was extracted by shaking with distilled water for $1 \mathrm{~h}$, vacuum filtering through a fiber filter with a pore space of $1.2 \mu \mathrm{m}$ (Fisher), and measurement with a liquid carbon and nitrogen analyzer Vario TOC cube (Elementar, Hanau, Germany). Dissolved total nitrogen (DTN), $\mathrm{NO}_{3}{ }^{-} \mathrm{N}$, and $\mathrm{NH}_{4}{ }^{+}-\mathrm{N}$ were extracted with $2 \mathrm{~mol} \mathrm{~L}^{-1} \mathrm{KCl}$, filtered, and measured using the San ++ continuous flow analyzer (Skalar, Breda, Netherlands). DON was calculated as $\mathrm{DON}=\mathrm{DTN}-\mathrm{NO}_{3}{ }^{-} \mathrm{N}$
- $\mathrm{NH}_{4}{ }^{+}-\mathrm{N}$. Values of all edaphic variables across the three elevations are shown in Table S1.

2.3 DNA extraction, library preparation, and amplicon sequencing

Total DNA was extracted under sterile conditions with a FastDNA Spin kit (MP Biomedicals, Santa Ana, CA). The extracted DNA was eluted in $70 \mu \mathrm{L}$ TE buffer and quantified with an ND1000 spectrophotometer (Thermo Fisher). The extracted DNA was amplified by targeting the bacterial V4-5 variable region of $16 \mathrm{~S}$ rDNA, a universal and reliable targeting zone, with primers F515: GTGCCAGCMGCCGCGG and R907: CCGTCAATTCMTTTRAGTTT equipped with unique identifier barcodes (Lane 1985). PCR amplification was performed in a $50-\mu \mathrm{L}$ reaction system containing $25 \mu \mathrm{L}$ Premix Taq DNA polymerase, $0.5 \mu \mathrm{L}$ forward primer $(20 \mu \mathrm{M})$, $0.5 \mu \mathrm{L}$ reverse primer $(20 \mu \mathrm{M}), 23 \mu \mathrm{L}$ double distilled water $\left(\mathrm{ddH}_{2} \mathrm{O}\right)$, and $1 \mu \mathrm{L}$ DNA template (20 ng total soil DNA). The conditions for PCR amplification were $94^{\circ} \mathrm{C}$ for $5 \mathrm{~min}$ and 35 cycles of $94^{\circ} \mathrm{C}$ for $30 \mathrm{~s}, 50^{\circ} \mathrm{C}$ for $30 \mathrm{~s}$, and $72^{\circ} \mathrm{C}$ for $30 \mathrm{~s}$. The PCR products were quality controlled to equimolar amounts before 250-bp paired-end sequencing on the Illumina MiSeq platform (Illumina, Inc., San Diego, CA, USA). All sequencing data associated with this study have been uploaded to the NCBI Sequence Read Archive (SRA) database under BioProject PRJNA597271.

\subsection{Bioinformatics}

We first merged the raw paired reads using FLASH software (Magoč et al., 2011). We then controlled the quality of the merged data using Cutadapt 1.9.1 to filter low-quality sequences and trim short sequences (Kuczynski et al., 2011; Martin, 2011). After quality filtering, we obtained highquality nucleotide sequences with min length $>200 \mathrm{bp}$ and Phred quality threshold $>29$. Chimeric sequences were detected and removed with the uchime3-denovo method as described in the VSEARCH user document (Rognes et al., 2016). The remaining sequences were clustered into $97 \%$ similar operational taxonomic units (OTUs) using the UCLUST algorithm in the QIIME 1.9.1 pipeline (Edgar, 2010). Representative sequences were selected and aligned with PyNAST (DeSantis et al., 2006). We used the assign_ taxonomy.py function of UCLUST to assign taxonomic information to each clustered OTU, and taxonomic information for each bacterial OTU was acquired from the Greengenes database (gg-13-8 release) (DeSantis et al., 2006). QIIME alpha_rarefaction.py and alpha_diversity.py scripts were used to assess alpha diversity, including OTU richness and Faith's phylogenetic diversity (Faith, 1992). All samples were rarefied to 32000 sequences per sample (the minimum number of original sequences in a sample) for subsequent analyses. 


\subsection{Co-occurrence network analyses}

To explore the biotic interactions within each elevation, we performed co-occurrence network analysis at the OTU level using the SPIEC-EASI (SParse InversE Covariance Estimation for Ecological Association Inference) network method (Kurtz et al., 2015). SPIEC-EASI is based on the inverse covariance matrix and has shown greater statistical power than other network methods; we implemented SPIEC-EASI using the function spiec.easi in the "SpiecEasi" $\mathrm{R}$ package (Layeghifard et al., 2017). To ensure that given bacterial OTUs were present in the majority of samples from each elevation, we filtered OTUs based on thresholds for occurrence (present in at least four plots) and abundance (at least $0.01 \%$ in total) using Seqtime's filterTaxonMatrix function. We then performed the entire "SpiecEasi" pipeline for data transformation, sparse inverse covariance estimation, and model selection among all 15 soil samples. The output was transformed with the adj2igraph script, and the resulting network files were imported into Cytoscape software to extract the sub-network at each elevation and calculate topological network properties (Shannon et al., 2003; Saito et al., 2012). In each of the three soil bacterial co-occurrence networks, topological features not only represented different network structures, but also indicated complex microbial interactions. At the whole cooccurrence network level, we selected some representative topological properties to reflect the network structure, including average number of neighbors, clustering coefficient, and network density. Average number of neighbors was calculated from degree scores, which represented the extent to which members were closely related to each other in the network. Clustering coefficients were based on connectedness among network members. Network density represented the proportion of actual connections relative to all potential connections. At the OTU level, network degree scores and betweenness centrality were critical topological parameters that reflected the position of species nodes in the co-occurrence network. We adopted the definition of network nodes based on the method proposed by Deng et al. (2012). Specifically, the nodes were classified into four categories: 1) network hubs with high within- $(\mathrm{Zi})$ and among-module $(\mathrm{Pi})$ connectivities $(\mathrm{Zi}>2.5 ; \mathrm{Pi}>0.62) ; 2)$ module hubs with only high withinmodule connectivities $(\mathrm{Zi}>2.5$; $\mathrm{Pi}<0.62)$; 3$)$ connectors with only high among-module connectivities $(\mathrm{Zi}<2.5 ; \mathrm{Pi}>0.62) ; 4)$ peripheral nodes with low within- and among-module connectivities $(\mathrm{Zi}<2.5 ; \mathrm{Pi}<0.62)$. Network plots were displayed using Gephi software (https://github.com/gephi/gephi).

\subsection{Statistical analyses}

Both edaphic variables and relative abundance data were subjected to normality tests before further analysis with the Shapiro-Wilk (SW) test using the shapiro.test function in the "stats" R package. Generalized variance-inflation factors (VIFs) were calculated and used to detect multicollinearity for linear models using the vif function in the "car" R package. Significant differences in relative abundance at the phylum/ subphylum level were tested by multiple comparisons using Duncan's test in the "EasyAovWlxPlot" $R$ package. Differences in soil physicochemical properties were also tested in the "EasyAovWlxPlot" package using the Kruskal-Wallis (KW) test. Correlations between diversity/abundance and edaphic variables were analyzed by the Spearman method using SPSS 20.0 (IBM, USA) for Microsoft Windows.

For the analyses of bacterial community composition, community composition data were first Hellinger-transformed with the decostand function, and the Bray-Curtis dissimilarity distance was then computed using the vegdist function in the "vegan" package. Environmental factor vectors were calculated using the envfit function in the "vegan" package. Distance-based linear model multivariate analysis (DISTLM) performed in DISTLM forward3 for Windows was used to eliminate autocorrelation of edaphic variables (Anderson, 2004). To measure the effects of elevation, NMDS was performed based on the Bray-Curtis distance using the metaMDS function in the "vegan" package. Permutational Multivariate Analysis of Variance (Adonis) was performed to partition the Bray-Curtis distance matrix by the factors of elevation and other environmental variables using the adonis function in the "vegan" R package.

\section{Results}

3.1 Bacterial community composition and diversity along a short elevational gradient

In total, 704431 nucleotide sequence counts were obtained from all soil samples and ranged from 32697 to 64930 per sample (median: 46 440; mean: 46 962). After quality control, 16473 unique OTUs based on a $97 \%$ similarity threshold were clustered and identified to the phylum level. The dominant phyla across the three elevations were Acidobacteria $(\sim 31.7 \%)$, Proteobacteria $(\sim 25.5 \%)$, Actinobacteria ( 9.0\%), Bacteroidetes ( 8.3\%), Verrucomicrobia $(\sim 7.4 \%)$, Crenarchaeota ( $5.5 \%)$, Planctomycetes $(\sim 3.6 \%)$, and Chloroflexi $(\sim 2.1 \%)$, which together accounted for more than $90 \%$ of the total abundance (Fig. 1A). The percentages of dominant bacterial phyla were similar between the hillfoot and the hillside. The Acidobacteria phylum dominated at the hillfoot and the hillside, whereas Proteobacteria prevailed at the hilltop. The relative abundance of Actinobacteria was significantly different among elevations (KW test: $P=0.008)$. The relative abundances of Alphaproteobacteria (KW test: $P=$ 0.004 ), Elusimicrobia (KW test: $P=0.024$ ), and Crenarchaeota (KW test: $P=0.012$ ), which were relatively low, also differed significantly among elevations. The above data are shown in Table S2.

We also studied community composition at the order level and explored the elevational differences in relative abundance 
A

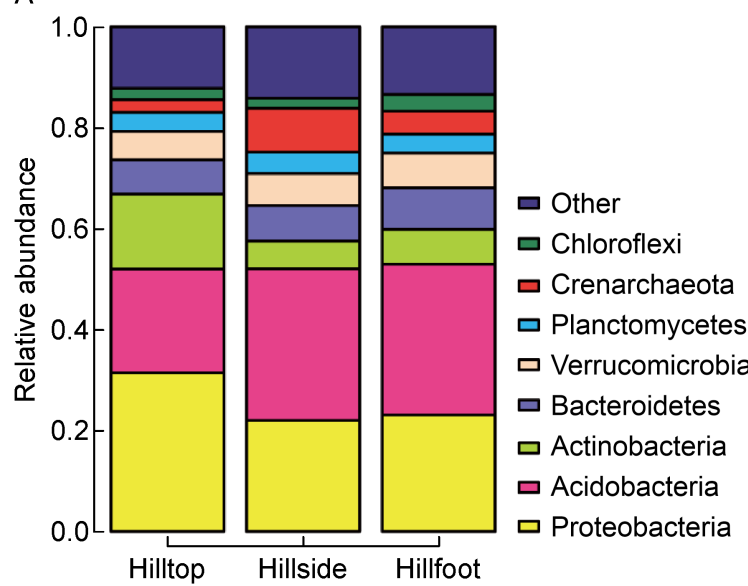

B

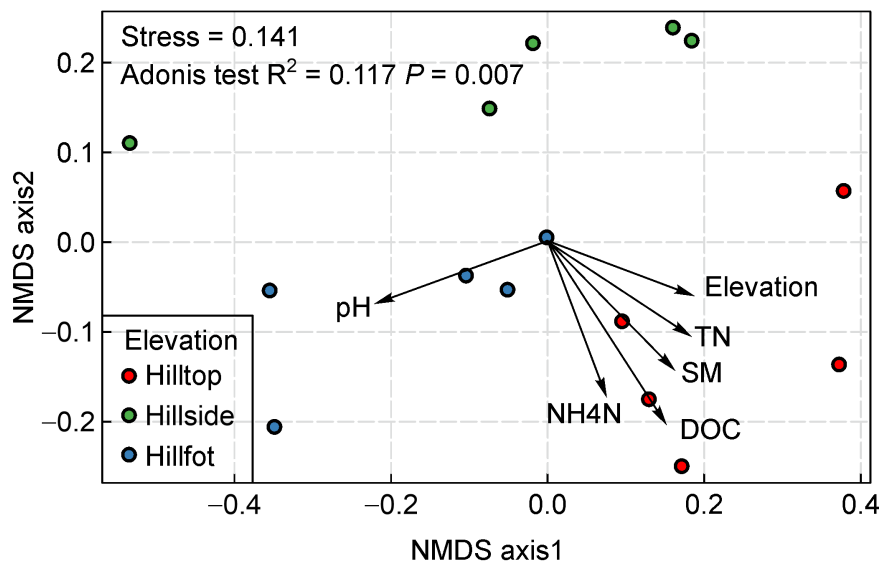

Fig. 1 (A) Relative abundance of dominant phyla in soils separated by their positions along an elevational gradient, based on the proportion of DNA sequences that could be classified at the phyla level. (B) Two-dimensional NMDS ordination of samples using the Bray-Curtis method and the 'envfit' function to fit significant environmental factors onto ordinations. Abbreviations for edaphic factors are given in Table 1.

of 24 bacterial orders that accounted for more than $70 \%$ of the total abundance (Fig. S2). The order Ellin6513 (Acidobacteria) had the highest average relative abundance. The relative abundances of Ellin6513 (Acidobacteria, KW test: $P=0.042$ ), Actinomycetales (Actinobacteria, KW test: $P=0.008$ ), Rhizobiales (Proteobacteria, KW test: $P=0.015)$, Rhodospirillales (Proteobacteria, KW test: $P=0.006$ ), Cenarchaeales (Crenarchaeota, KW test: $P=0.018$ ), Thermogemmatisporales (Chloroflexi, KW test: $P=0.025$ ), Nitrososphaerales (Crenarchaeota, KW test: $P=0.01$ ), and Solirubrobacterales (Actinobacteria, KW test: $P=0.023$ ) differed significantly among elevations (Table S3). Bacterial community composition was also significantly different among elevations (Fig. 1B; Table S4), indicating the presence of distinct elevational variation even along a small-scale elevational gradient. Nevertheless, we did not find any significant differences in phylogenetic diversity or OTU richness of the bacterial community among the three elevations (Fig. S3).

\subsection{Effects of environmental factors on bacterial community structure}

SM, TC, and $\mathrm{NH}_{4}{ }^{+}-\mathrm{N}$ data that did not follow a normal distribution were log-transformed (Table S5). We also removed TC from the analysis to avoid issues of multicollinearity (Table S6). The remaining environmental factors were used in subsequent analyses. The distribution pattern of the soil bacterial community (NMDS plot) and its driving factors (envfit) are shown in Fig. 1B. Multiple comparative analysis showed that edaphic variables changed significantly with elevation, with the exception of soil $\mathrm{pH}$ (Table S1). We performed an Adonis test based on analysis of variance to calculate the relative contributions of environmental factors in the variation of bacterial community composition. The Adonis results showed that soil $\mathrm{pH}, \mathrm{DOC}, \mathrm{TN}$, elevation, $\mathrm{SM}$, and $\mathrm{NO}_{3}{ }^{-} \mathrm{N}$ were significantly correlated with bacterial community composition (Table S7). DistLM analysis using linear modeling
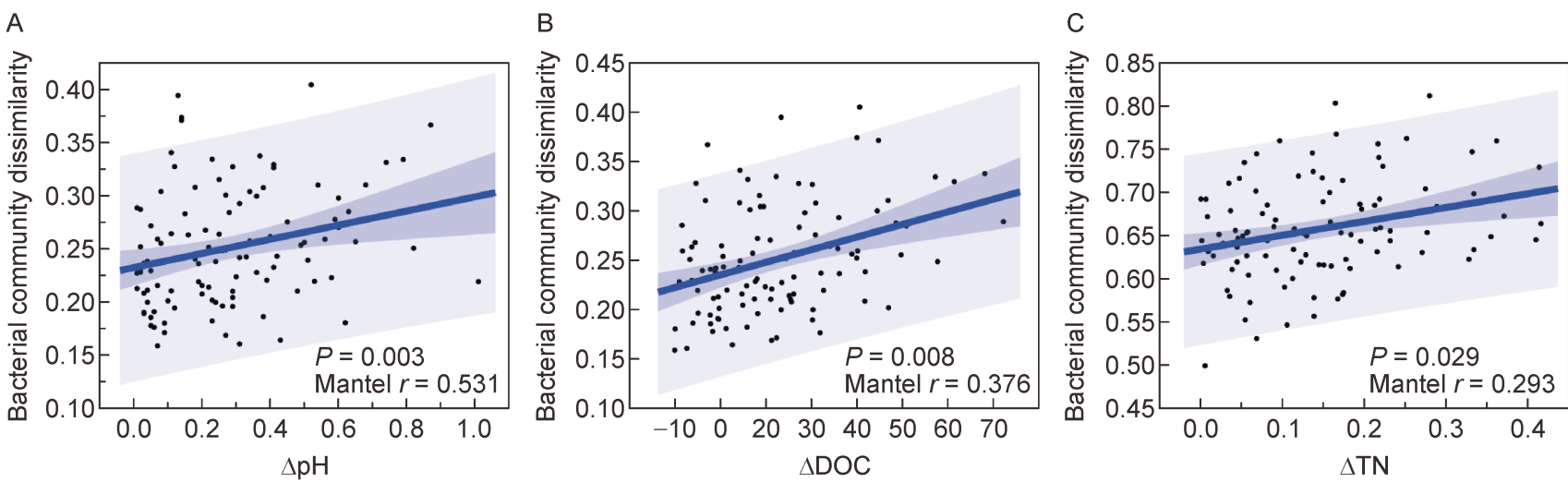

Fig. 2 Relationships between bacterial community dissimilarity and soil $\mathrm{pH}(\mathrm{A}), \mathrm{DOC}(\mathrm{B})$, and $\mathrm{TN}(\mathrm{C}) . \triangle$ indicates the difference in $\mathrm{pH}$, $\mathrm{DOC}$, and TN between paired elevations. Dissimilarity was calculated by Bray-Curtis distance. 
based on matrices was used to explore the effects of environmental factors on bacterial community composition. When variables were fitted individually, DistLM results confirmed that soil $\mathrm{pH}, \mathrm{TN}$, and DOC were the most influential edaphic drivers of soil bacterial community composition. These factors explained $7.55 \%, 7.52 \%$, and $7.46 \%$ of the variation among elevations, respectively (Table 1). Elevation also showed a significant contribution to the variation in bacterial community composition. When forward selection variables were fitted partially and sequentially, only soil $\mathrm{pH}$ played a significant role in the variation of bacterial community composition. We then calculated the DistLM without soil $\mathrm{pH}$, which left only elevation as a significant factor (Table 1). In addition, regressions between the Bray-Curtis dissimilarity of the bacterial community and differences in soil $\mathrm{pH}, \mathrm{DOC}$, and TN were performed, revealing that soil $\mathrm{pH}, \mathrm{DOC}$, and TN had significant explanatory power for bacterial community composition (Fig. 2). Finally, correlation analyses between environmental factors and diversity were performed. Only soil $\mathrm{pH}$ was correlated with phylogenetic diversity $(r=0.549 ; P=0.034)$ and OTU richness $(r=0.505 ; P=0.055$; Table S8).

At the phylum level, the relative abundances of Verrucomicrobia and Deltaproteobacteria were significantly positively correlated with soil $\mathrm{pH}$, whereas that of Gammaproteobacteria was negatively correlated with $\mathrm{pH}$. The relative abundances of Alphaproteobacteria were significantly and positively correlated with DOC. The relative abundances of Alphaproteobacteria and Gammaproteobacteria were significantly and

Table 1 Distance-based multivariate linear model (DistLM) for bacterial community composition, calculated by Bray-Curtis distance.

\begin{tabular}{|c|c|c|c|c|}
\hline Variable $^{a}$ & $\%$ Var. $^{\mathrm{b}}$ & Pseudo-F & $P^{c}$ & Cumulative \\
\hline \multicolumn{5}{|c|}{ Variables fitted individually } \\
\hline $\mathrm{pH}$ & 0.0755 & 1.061 & 0.0004 & I \\
\hline Elevation & 0.0752 & 1.0578 & 0.0006 & I \\
\hline TN & 0.0752 & 1.0564 & 0.0016 & I \\
\hline DOC & 0.0746 & 1.0481 & 0.0034 & I \\
\hline SM & 0.074 & 1.0388 & 0.0104 & I \\
\hline $\mathrm{NO}_{3}{ }^{-}-\mathrm{N}$ & 0.0737 & 1.0349 & 0.0186 & l \\
\hline $\mathrm{NH}_{4}^{+}-\mathrm{N}$ & 0.073 & 1.0243 & 0.0492 & I \\
\hline DON & 0.0711 & 0.9948 & 0.588 & 1 \\
\hline \multicolumn{5}{|c|}{ Variables fitted sequentially } \\
\hline $\mathrm{pH}$ & 0.0755 & 1.061 & 0.0004 & 0.0755 \\
\hline Elevation & 0.0738 & 1.0416 & 0.495 & 0.1493 \\
\hline DON & 0.0731 & 1.0337 & 0.5162 & 0.2224 \\
\hline TN & 0.0728 & 1.0334 & 0.5138 & 0.2952 \\
\hline $\mathrm{NH}_{4}^{+}-\mathrm{N}$ & 0.0718 & 1.0211 & 0.5072 & 0.367 \\
\hline SM & 0.0707 & 1.0062 & 0.5222 & 0.4377 \\
\hline $\mathrm{NO}_{3}{ }^{-}-\mathrm{N}$ & 0.0705 & 1.0034 & 0.5094 & 0.5082 \\
\hline DON & 0.0701 & 0.9968 & 0.5018 & 0.5783 \\
\hline \multicolumn{5}{|c|}{ Variables fitted sequentially without soil $\mathrm{pH}$} \\
\hline Elevation & 0.0752 & 1.0578 & 0.0006 & 0.0752 \\
\hline DOC & 0.0733 & 1.0333 & 0.5318 & 0.1486 \\
\hline TN & 0.0727 & 1.0273 & 0.5264 & 0.2213 \\
\hline $\mathrm{NH}_{4}{ }^{+}-\mathrm{N}$ & 0.0718 & 1.0153 & 0.5522 & 0.2931 \\
\hline DON & 0.0713 & 1.0092 & 0.5236 & 0.3643 \\
\hline $\mathrm{NO}_{3}{ }^{-}-\mathrm{N}$ & 0.0712 & 1.0086 & 0.509 & 0.4355 \\
\hline SM & 0.0706 & 1.0013 & 0.5114 & 0.5062 \\
\hline
\end{tabular}

a SM, soil moisture. TN, total N. DOC, dissolved organic carbon. DON, dissolved organic nitrogen.

b The percentage of variance explained by a given variable is abbreviated as "\% Var."

${ }^{\mathrm{c}}$ Bold values indicate significant correlations $(P<0.05)$. 
positively correlated with TN, whereas Chloroflexi abundance was negatively correlated with TN. Only Alphaproteobacteria was correlated with elevation. Additional information on edaphic variables and dominant phyla/subphyla is presented in Table S9 and Fig. S4. The above analyses (envfit, Adonis, and DistLM) also supported the hypothesis that bacterial community distribution along small-scale elevational gradients is driven by soil $\mathrm{pH}$ and elevation.

\subsection{Co-occurrence networks of soil bacterial communities}

The species co-occurrence networks of soil bacterial communities in all elevations showed subtle differences at the network and node levels (Fig. S5). At the network level, network topological properties, including the average number of neighbors, the clustering coefficient, and positive/negative links, differed among the three elevations (Table S10). The average number of neighbors was highest but the clustering coefficient was lowest in the hilltop network. Positive correlations were predominant at all elevations and increased with increasing elevation. Other network topology features are presented in Table S10. At the OTU level, the hilltop network had the highest mean degree scores, and the hillside network had the lowest scores (Fig. 3); the hillside network mean degree scores differed significantly from those of the hilltop and hillfoot networks (Table S11). Mean betweenness centrality was highest in the hillside network and lowest in the hilltop network, and the betweenness centrality of the hillside network differed significantly from those of the other networks.

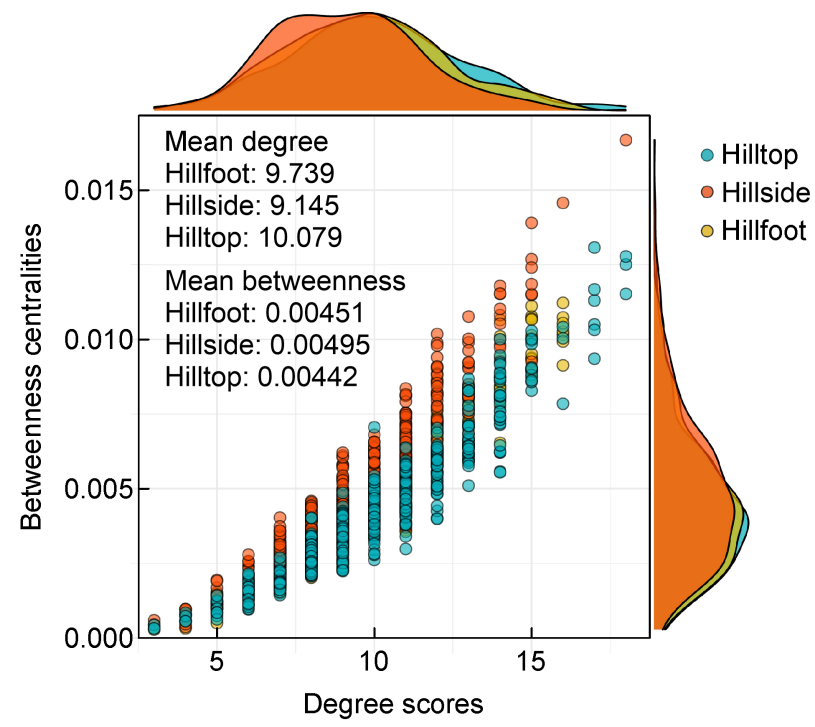

Fig. 3 Relationships between degree scores and betweenness centralities, classified according to elevations (cyan: Hilltop; orange: Hillside; yellow: Hillfoot).

The $\mathrm{Zi}-\mathrm{Pi}$ relationship analysis showed that bacterial phenotypes involved in co-occurrence networks played different roles in the networks (Fig. 4). Most nodes were peripheral at all elevations, followed by connectors, module hubs, and network hubs; the module hubs and network hubs were regarded as keystone species. We identified the phenotype belonging to Phylum Acidobacteria Family Koribacteraceae (OTU 291945) and the phenotype belonging to Phylum Proteobacteria Order Ellin329 (denovo124438) as network hubs in the hillfoot and hillside networks, respectively, but there was no network hub in the hilltop network. We also identified multiple module hubs in the networks (8 nodes in the hillfoot network, 3 nodes in the hillside network, and 5 nodes in the hilltop network), and these belonged mainly to Proteobacteria and Acidobacteria (Table S12).

Correlation analysis highlighted the relationships between environmental factors and relative abundance of hubs (Fig. 5; Table S13). Soil moisture was significantly correlated with denovo172651 (Module hub, Crenarchaeota), denovo65042 (Module hub, Proteobacteria), denovo250717 (Module hub, Proteobacteria), and denovo58422 (Module hub, Proteobacteria). The module hubs 248716 (Acidobacteria), denovo172651 (Crenarchaeota), denovo95684 (Actinobacteria), denovo65042 (Proteobacteria), and denovo250717 (Proteobacteria) showed significant correlations with TN. DOC was significantly correlated with denovo58422 (Module hub, Proteobacteria) from Order Rhizobiales. Elevation was significantly correlated with module hubs typified by the Proteobacteria and Acidobacteria phyla, including 248716, denovo172651, denovo65042, and denovo250717. Additional correlations are presented in Table S13 and Fig. 5.

\section{Discussion}

4.1 Bacterial community composition differed along a smallscale elevational gradient, whereas diversity did not

We observed strong partitioning of bacterial community composition but no significant variation in bacterial diversity along the small-scale elevational gradient on Zijin mountain. The lack of significant variation in diversity was consistent with many previous studies (Fierer et al., 2011; Shen et al., 2013; Xu et al., 2014; Fig. S4). However, several studies focusing on small scales have also reported significant changes in diversity with elevation; for example, Shen et al. (2015) found that diversity decreased with increasing elevation along a 500-m gradient on Changbai mountain. The conflicting diversity patterns may be caused by climatic factors (Lanzen et al., 2016; Wang and Soininen, 2017) and different levels of niche-based environmental filtering related to soil $\mathrm{C} / \mathrm{N}$ content (Okie et al., 2015; Li et al., 2020). It is difficult to judge the relative contributions of climate and environmental filtering along such a short gradient due to the homogeneity of these factors. Microbial distributions along small-scale elevational gradients in urban landscape ecosystems may be more relevant to human activities than those in natural ecosystems (Bai et al., 2017). Combining information on microbial distributions with the health-related microbiome (including 
probiotics and pathogens) might deepen our understanding of associations between microbial distribution and public health in the urban landscape (Xu et al., 2014; Reese et al., 2016). In the future, the integration of studies with a large range of niche-based differences, such as meta-analyses or continental-scale surveys, could be an appropriate way to explore related issues.

The beta diversity results showed significant effects of elevation on bacterial distribution along a small-scale elevational gradient. The apparent shifts in relative abundance of key phyla/subphyla, including an increase in Proteobacteria and Actinobacteria and a decrease in Acidobacteria and Bacteroidetes with increasing elevation, may be an essential reason for the strong partitioning of community composition.
In fact, strong partitioning of community composition is usually associated with soil functions (Crowther et al., 2019). For example, Gammaproteobacteria adapt to more acidic habitats and perform the soil function of $\mathrm{C}$ cycling; here, their abundance was correlated with soil $\mathrm{pH}(r=0.55 ; P=0.034)$ and carbon $(r=0.542 ; P=0.037)$. This may reflect the fact that forests at high elevations have recalcitrant SOC and reduced litter inputs because of the large fraction of needles (Qu et al., 2020). Therefore, the ecological niches of Gammaproteobacteria, which are acidophilic organisms, may be related to conifer forest types. The present results imply that soil functions along small-scale elevational gradients may primarily reflect the activities of the abovementioned phyla whose abundances change with elevation, although this conjecture
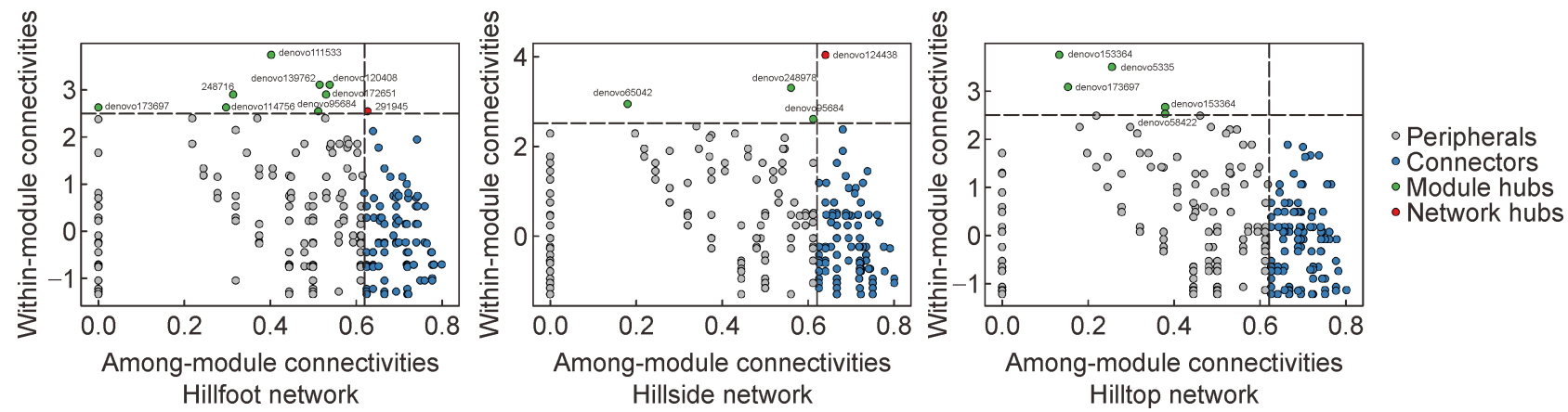

Fig. 4 Zi-Pi plots showing distributions of OTUs based on their topological roles in Hillfoot, Hillside, and Hilltop networks. Sub-data sets consisting of five samples were extracted from the total network. The threshold values of $\mathrm{Zi}$ and $\mathrm{Pi}$ used to categorize OTUs were 2.5 and 0.62 , respectively.

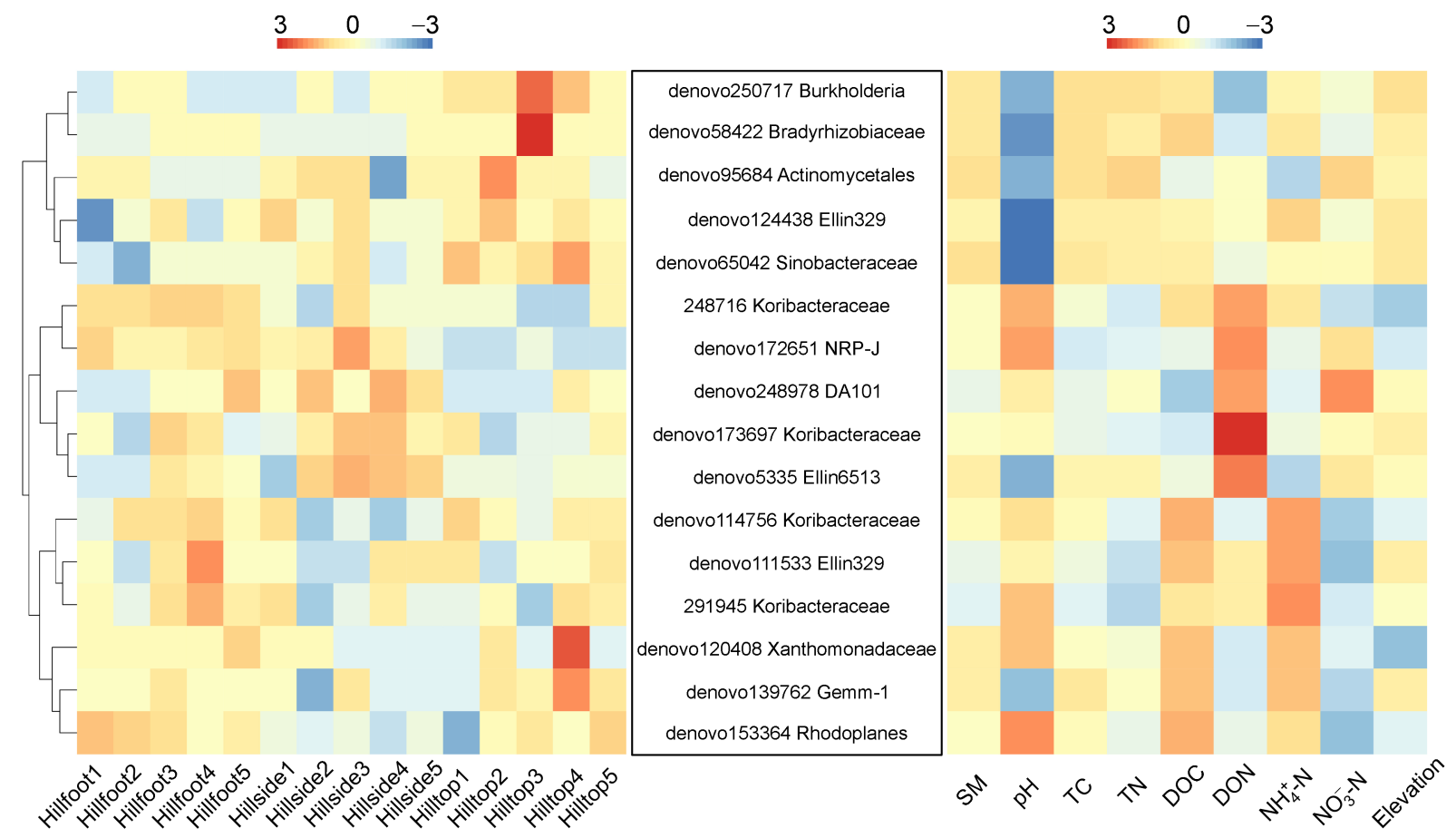

Fig. 5 Heat map showing the relative abundance of keystone species. The left heat map shows hub proportions sorted by sampling sites, and the right heat map shows the correlations between relative abundance of hubs and variables based on the Spearman method. The hierarchical dendrogram on the left was clustered based on the relative abundance of hubs. 
remains to be verified with more data and experiments in the future.

4.2 Soil $\mathrm{pH}$ and elevation were associated with variations in bacterial community composition

The mechanisms that drive community composition along small-scale elevational gradients in urban landscape ecosystems have gained increasing interest. Because of the low environmental heterogeneity and urban effects in such systems, the differences in driving factors between largeand small-scale studies are worthy of attention. In our study, bacterial community composition was significantly correlated with the edaphic variables of soil $\mathrm{pH}, \mathrm{TN}$, and DOC, as well as the environmental factor of elevation (Table 1). The responses of some specific phyla to $\mathrm{pH}, \mathrm{DOC}$, and TN were similar to those reported in other studies (Jones et al., 2009; Chu et al., 2010; Rousk et al., 2010). For instance, the relative abundance of Verrucomicrobia related to $C$ mineralization and methanotrophy increased with higher $\mathrm{pH}$, whereas that of Gammaproteobacteria, which also carries out the function of $\mathrm{C}$ cycling, decreased. These results also imply that although total community composition was significantly affected by soil $\mathrm{pH}, \mathrm{DOC}$, and TN, specific phyla responded to these factors in different ways. For example, the abundance of Alphaproteobacteria, which perform $\mathrm{C}$ and $\mathrm{N}$ fixation, was significantly related to the content of soil $\mathrm{C}$ and $\mathrm{N}$ but not to soil $\mathrm{pH}$ (Table S9). Community composition was also affected by elevation as a comprehensive factor, including scale effects (limitation dispersal) and environment filtering (niche selection). In addition, strong correlations between environmental factors and rare phyla with low abundances, such as Chloroflexi, indicated that the relative abundance of rare phyla was also markedly influenced by environmental factors. The overriding importance of $\mathrm{pH}$ in the regulation of soil bacterial community structure has been widely reported across both vertical and horizontal scales (Fierer and Jackson, 2006; Griffiths et al., 2011; Shen et al., 2013; Shen et al., 2019). However, these studies were performed across large scales. In this study, we found that soil $\mathrm{pH}$ was still the most important factor affecting bacterial distribution along a small-scale elevational gradient. A change of one soil $\mathrm{pH}$ unit implies a 10-fold difference in $\mathrm{H}^{+}$ concentration, and small changes in soil $\mathrm{pH}$ are therefore very large changes in $\mathrm{H}^{+}$concentration. Klimek et al. (2015) showed that even a small variation in soil $\mathrm{pH}$ affected bacterial catabolic activity. Vegetation may also affect the soil microbial community through modifications of root exudates and soil properties (Trivedi et al., 2020). Although we did not survey plant communities at the time of sampling, previous work has shown that different vegetation types related to forest succession are associated with different soil bacterial communities in Zijin Mountain (Qu et al., 2020). As mentioned above, future studies of driving mechanisms along smallscale elevational gradients in urban areas will extend beyond soil $\mathrm{pH}$ and elevation to assess the effects of plants, climate, urbanization, and other factors.
4.3 Strong partitioning of network topology along a small-scale elevational gradient

Our study addressed the question of whether co-occurrence network patterns differ between elevations at two levels: network and node topology. Network topology among different elevations differed in the frequency, composition, and distribution of interactions (Fig. 3; Table S10). At the network level, the hilltop network had a higher average number of neighbors, indicating closer interactions among bacterial communities at this elevation. However, the hillside network had a larger clustering coefficient within bacterial groups than other networks. The more robust clustering coefficient may reflect more active microbial interactions shaping network topological features (Röttjers and Faust, 2018). At the OTU level, the OTUs typifying the hilltop elevation had the lowest betweenness centrality, indicating that this microbial community was less affected by interactions. The degree score is described as the number of direct co-occurrence interactions of a specific species (Greenblum et al., 2012). The mean degree scores of whole network nodes were highest in the hilltop network, suggesting a greater degree of association among its members.

Recent studies have reported distinct microbial interaction patterns. For instance, Ma et al. (2016) found that microorganisms in northern regions had closer relationships compared with those in southern regions, and microbial networks in soybean fields from southern China had more interactions than those from northern China (Zhang et al., 2018). According to the study of Li et al. (2018), differentiation of ecological niches caused by elevation and key edaphic variables (e.g., pH, DOC, and TN) may be an essential cause of network topological divergence. Li et al. (2020) proposed the niche differentiation theory that the stronger the differentiation of ecological niches, the weaker the microbial interactions. In this study, the hillop network exhibited closer and more complicated network relationships, potentially implying that it had lower ecological niche differentiation and less variable environmental conditions (Table S1). In addition to niche differentiation, variation in soil $\mathrm{pH}$ may be another important factor in network divergence ( $\mathrm{Li}$ et al., 2018). In summary, the co-occurrence network properties of bacterial communities at all elevations were distinct.

\subsection{Keystone species differed between elevations}

Keystone species exert considerable influence on other network members and play critical roles in microbial interactions; they are identified based on natural connectivities within and among modules (Ma et al., 2016; Banerjee et al., 2018). The composition of keystone species also differed significantly among elevations, addressing our second question (Fig. 4; Table S12). The most dominant keystone species belonged to Acidobacteria and Proteobacteria at the three elevations. It was consistent between the dominant phyla and network hubs, the potential relationships awaiting to uncover 
in the future. Hubs generally have a longer evolutionary history and coordinate or dominate ecological processes in the soil ecosystem (Barabási, 2009; Berry and Widder, 2014). For example, within the Proteobacteria, hubs from the order Rhizobiales are tightly linked with nitrogen-fixing capabilities around plant roots (Brown et al., 2012; Fan et al., 2017). In the present study, however, almost all the hubs belonged to Acidobacteria and Proteobacteria, whose specific ecological functions are still unknown. Similarly, hubs included relatively unknown groups that are highly abundant in the plant rhizosphere and are correlated with C cycling functions (Yuan et al., 2015). We also analyzed the relationships between environmental factors and keystone species. We found that module hub denovo65042 from the phylum Proteobacteria and family Sinobacteraceae, which is related to organic matter degradation and the use of carbon sources, was significantly correlated with soil TC and DOC, implying its association with soil C functions (Du et al., 2017). However, although the order Rhodoplanes was previously found to be tightly linked to plant roots (Zhalnina et al., 2015; Gkarmiri et al., 2017), it showed no correlations with any measured environmental variables. Future work focusing on uncultured species, such as the provisional order Ellin6513, will be fundamental for understanding the role of these hubs in urban forest soil ecosystems.

Co-occurrence network analysis provides a valuable tool for understanding microbial interactions; however, this tool is recognized as a generator of new hypotheses rather than a means of establishing firm conclusions (Röttjers and Faust, 2018). For example, whether the concept of social networks is directly applicable to microbial ecological networks has yet to be conclusively demonstrated. For now, the integration of network analytics into microbial ecology is a major methodological breakthrough. Nevertheless, experimental validation is critical for studies that report new phenomena, as there are a number of remaining unresolved issues (Lima-Mendez et al. 2015; Weiss et al. 2016).

\section{Conclusions}

Numerous studies have focused on the distribution of soil microbes along large-scale elevational gradients, but smallscale mountains located in urban landscape ecosystems have been overlooked. In this study, there were significant differences in microbial community composition and specific dominant bacterial phyla (e.g., Proteobacteria, Actinobacteria) along a small-scale elevational gradient, but there were no significant differences in diversity. Soil $\mathrm{pH}$ and elevation were key factors that drove the soil bacterial distribution. Network topological properties and composition of keystone species differed among elevations, which indicated the complex interspecific relationships within the bacterial communities. Taken together, these results demonstrate that the soil bacterial distribution differs along a small-scale elevational gradient on a publicly accessible urban mountain. This unique ecosystem type has been neglected in previous microbial ecology studies. The current study provides crucial insight into changes in microbial co-occurrence patterns with elevation and suggests some potential soil ecological functions of this unique ecosystem.

\section{Acknowledgments}

We are grateful to Kunkun Fan, Hongfei Wang, Xingjia Xiang, Kaoping Zhang, Yingying Ni, Dan He, Ruibo Sun, and Yuntao Li for assistance in soil sampling and laboratory analyses. This work was funded by the Strategic Priority Research Program of the Chinese Academy of Sciences (XDB15010101), the National Natural Science Foundation of China (41907039), and the China Biodiversity Observation Networks (Sino BON). The authors declare that they have no competing interests.

\section{Electronic supplementary material}

Supplementary material is available in the online version of this article at https://doi.org/10.1007/s42832-021-0122-2 and is accessible for authorized users.

\section{References}

Anderson, M., 2004. DISTLM v. 5: a FORTRAN computer program to calculate a distance-based multivariate analysis for a linear model. Department of Statistics, University of Auckland, New Zealand 10, 2016.

Bai, X., McPhearson, T., Cleugh, H., Nagendra, H., Tong, X., Zhu, T., Zhu, Y.G., 2017. Linking urbanization and the environment: conceptual and empirical advances. Annual Review of Environment and Resources 42, 215-240.

Banerjee, S., Schlaeppi, K., van der Heijden, M.G., 2018. Keystone taxa as drivers of microbiome structure and functioning. Nature Reviews. Microbiology 16, 567-576.

Barabási, A.L., 2009. Scale-free networks: a decade and beyond. Science 325, 412-413.

Berry, D., Widder, S., 2014. Deciphering microbial interactions and detecting keystone species with co-occurrence networks. Frontiers in Microbiology 5, 219.

Brown, P.J., de Pedro, M.A., Kysela, D.T., Van der Henst, C., Kim, J., De Bolle, X., Fuqua, C., Brun, Y.V., 2012. Polar growth in the Alphaproteobacterial order Rhizobiales. Proceedings of the National Academy of Sciences of the United States of America 109, 1697-1701.

Chaffron, S., Rehrauer, H., Pernthaler, J., von Mering, C., 2010. A global network of coexisting microbes from environmental and whole-genome sequence data. Genome Research 20, 947-959.

Chu, H., Fierer, N., Lauber, C.L., Caporaso, J.G., Knight, R., Grogan, P., 2010. Soil bacterial diversity in the Arctic is not fundamentally different from that found in other biomes. Environmental Microbiology 12, 2998-3006.

Chu, H., Gao, G.F., Ma, Y., Fan, K., Delgado-Baquerizo, M., 2020. Soil Microbial Biogeography in a Changing World: Recent Advances and Future Perspectives. mSystems 5, e00803-e00819. 
Crowther, T.W., van den Hoogen, J., Wan, J., Mayes, M.A., Keiser, A. D., Mo, L., Averill, C., Maynard, D.S., 2019. The global soil community and its influence on biogeochemistry. Science 365 , 772.

Deng, Y., Jiang, Y.H., Yang, Y., He, Z., Luo, F., Zhou, J., 2012. Molecular ecological network analyses. BMC Bioinformatics 13, 113.

DeSantis, T., Hugenholtz, P., Keller, K., Brodie, E., Larsen, N., Piceno, Y., Phan, R., Andersen, G.L., 2006. NAST: a multiple sequence alignment server for comparative analysis of $16 \mathrm{~S}$ rRNA genes. Nucleic Acids Research 34, W394-W399.

DeSantis, T.Z., Hugenholtz, P., Larsen, N., Rojas, M., Brodie, E.L., Keller, K., Huber, T., Dalevi, D., Hu, P., Andersen, G.L., 2006. Greengenes, a chimera-checked 16S rRNA gene database and workbench compatible with ARB. Applied and Environmental Microbiology 72, 5069-5072.

Dong, L., Xu, H., Ju, F., Liu, S., Wan, Z., 2011. Plant diversity and its conservation strategies in Zijin Mountain National Forest Park in Nanjing. Journal of Jiangsu Forestry Science \& Technology. 38, 30-35.

Du, S., Yu, M., Liu, F., Xiao, L., Zhang, H., Tao, J., Gu, W., Gu, J., Chen, X., 2017. Effect of facility management regimes on soil bacterial diversity and community structure. Chinese Journal of Eco-Agriculture 25, 1615-1625.

Edgar, R.C., 2010. Search and clustering orders of magnitude faster than BLAST. Bioinformatics (Oxford, England) 26, 2460-2461.

Faith, D.P., 1992. Conservation evaluation and phylogenetic diversity. Biological Conservation 61, 1-10.

Fan, K., Cardona, C., Li, Y., Shi, Y., Xiang, X., Shen, C., Wang, H., Gilbert, J.A., Chu, H., 2017. Rhizosphere-associated bacterial network structure and spatial distribution differ significantly from bulk soil in wheat crop fields. Soil Biology \& Biochemistry 113, 275-284.

Fan, K., Delgado-Baquerizo, M., Guo, X., Wang, D., Wu, Y., Zhu, M., Yu, W., Yao, H., Zhu, Y.G., Chu, H., 2019. Suppressed N fixation and diazotrophs after four decades of fertilization. Microbiome 7 , 143.

Farrer, E.C., Porazinska, D.L., Spasojevic, M.J., King, A.J., Bueno De Mesquita, C.P., Sartwell, S.A., Smith, J.G., White, C.T., Schmidt, S. K., Suding, K.N., 2019. Soil microbial networks shift across a highelevation successional gradient. Frontiers in Microbiology 10, 2887.

Faust, K., Raes, J., 2012. Microbial interactions: from networks to models. Nature Reviews Microbiology 10, 538-550.

Fierer, N., Jackson, R.B., 2006. The diversity and biogeography of soil bacterial communities. Proceedings of the National Academy of Sciences of the United States of America 103, 626-631.

Fierer, N., McCain, C.M., Meir, P., Zimmermann, M., Rapp, J.M., Silman, M.R., Knight, R., 2011. Microbes do not follow the elevational diversity patterns of plants and animals. Ecology 92, 797-804.

Gkarmiri, K., Mahmood, S., Ekblad, A., Alstrom, S., Hogberg, N., Finlay, R., 2017. Identifying the active microbiome associated with roots and rhizosphere soil of oilseed rape. Applied and Environmental Microbiology 83, e01938-e17.
Greenblum, S., Turnbaugh, P.J., Borenstein, E., 2012. Metagenomic systems biology of the human gut microbiome reveals topological shifts associated with obesity and inflammatory bowel disease. Proceedings of the National Academy of Sciences of the United States of America 109, 594-599.

Griffiths, R.I., Thomson, B.C., James, P., Bell, T., Bailey, M., Whiteley, A.S., 2011. The bacterial biogeography of British soils. Environmental Microbiology 13, 1642-1654.

Huot, H., Joyner, J., Cordoba, A., Shaw, R.K., Wilson, M.A., Walker, R., Muth, T.R., Cheng, Z.Q., 2017. Characterizing urban soils in New York City: profile properties and bacterial communities. Journal of Soils and Sediments 17, 393-407.

Jones, R.T., Robeson, M.S., Lauber, C.L., Hamady, M., Knight, R., Fierer, N., 2009. A comprehensive survey of soil acidobacterial diversity using pyrosequencing and clone library analyses. ISME Journal 3, 442-453.

Klimek, B., Niklińska, M., Jaźwa, M., Tarasek, A., Tekielak, I., Musielok, $Ł$., 2015. Covariation of soil bacteria functional diversity and vegetation diversity along an altitudinal climatic gradient in the Western Carpathians. Pedobiologia 58, 105-112.

Kuczynski, J., Stombaugh, J., Walters, W.A., González, A., Caporaso, J.G., Knight, R., 2011. Using QIIME to analyze 16S rRNA gene sequences from microbial communities. Current Protocols in Bioinformatics 10, 17.

Kurtz, Z.D., Müller, C.L., Miraldi, E.R., Littman, D.R., Blaser, M.J., Bonneau, R.A., 2015. Sparse and compositionally robust inference of microbial ecological networks. PLoS Computational Biology 11, e1004226.

Ladau, J., Shi, Y., Jing, X., He, J.S., Chen, L., Lin, X., Fierer, N., Gilbert, J.A., Pollard, K.S., Chu, H., 2018. Existing climate change will lead to pronounced shifts in the diversity of soil prokaryotes. mSystems 3, e00167-e18.

Lane, D.J., Pace, B., Olsen, G.J., Stahl, D.A., Sogin, M.L., Pace, N.R., 1985. Rapid determination of $16 \mathrm{~S}$ ribosomal RNA sequences for phylogenetic analyses. Proceedings of the National Academy of Sciences of the United States of America 82, 6955-6959.

Lanzen, A., Epelde, L., Blanco, F., Martin, I., Artetxe, U., Garbisu, C., 2016. Multi-targeted metagenetic analysis of the influence of climate and environmental parameters on soil microbial communities along an elevational gradient. Scientific Reports 6, 28257.

Layeghifard, M., Hwang, D.M., Guttman, D.S., 2017. Disentangling Interactions in the Microbiome: A Network Perspective. Trends in Microbiology 25, 217-228.

Li, G., Sun, G.X., Ren, Y., Luo, X.S., Zhu, Y.G., 2018. Urban soil and human health: a review. European Journal of Soil Science 69, 196-215.

Li, J., Li, C., Kou, Y., Yao, M., He, Z., Li, X., 2020. Distinct mechanisms shape soil bacterial and fungal co-occurrence networks in a mountain ecosystem. FEMS Microbiology Ecology 96, fiaa030.

Li, J., Shen, Z., Li, C., Kou, Y., Wang, Y., Tu, B., Zhang, S., Li, X., 2018. Stair-step pattern of soil bacterial diversity mainly driven by $\mathrm{pH}$ and vegetation types along the elevational gradients of Gongga Mountain, China. Frontiers in Microbiology 9, 569.

Lima-Mendez, G., Faust, K., Henry, N., Decelle, J., Colin, S., Carcillo, F., Chaffron, S., Ignacio-Espinosa, J.C., Roux, S., Vincent, F., 
Bittner, L., Darzi, Y., Wang, J., Audic, S., Berline, L., Bontempi, G., Cabello, A.M., Coppola, L., Cornejo-Castillo, F.M., d'Ovidio, F., De Meester, L., Ferrera, I., Garet-Delmas, M.J., Guidi, L., Lara, E., Pesant, S., Royo-Llonch, M., Salazar, G., Sanchez, P., Sebastian, M., Souffreau, C., Dimier, C., Picheral, M., Searson, S., KandelsLewis, S., Gorsky, G., Not, F., Ogata, H., Speich, S., Stemmann, L., Weissenbach, J., Wincker, P., Acinas, S.G., Sunagawa, S., Bork, P., Sullivan, M.B., Karsenti, E., Bowler, C., de Vargas, C., Raes, J., 2015. Determinants of community structure in the global plankton interactome. Science 348, 1262073.

Ma, B., Wang, H., Dsouza, M., Lou, J., He, Y., Dai, Z., Brookes, P.C., Xu, J., Gilbert, J.A., 2016. Geographic patterns of co-occurrence network topological features for soil microbiota at continental scale in eastern China. ISME Journal 10, 1891-1901.

Magoč, T., Salzberg, S.L., 2011. FLASH: fast length adjustment of short reads to improve genome assemblies. Bioinformatics (Oxford, England) 27, 2957-2963.

Martin, M., 2011. Cutadapt removes adapter sequences from highthroughput sequencing reads. EMBnet.Journal 17, 10-12.

Okie, J.G., Van Horn, D.J., Storch, D., Barrett, J.E., Gooseff, M.N., Kopsova, L., Takacs-Vesbach, C.D., 2015. Niche and metabolic principles explain patterns of diversity and distribution: theory and a case study with soil bacterial communities. Proceedings. Biological Sciences 282, 9.

Qu, Z.L., Liu, B., Ma, Y., Xu, J., Sun, H., 2020. The response of the soil bacterial community and function to forest succession caused by forest disease. Functional Ecology 34, 2548-2559.

Ramirez, K.S., Leff, J.W., Barberan, A., Bates, S.T., Betley, J., Crowther, T.W., Kelly, E.F., Oldfield, E.E., Shaw, E.A., Steenbock, C., Bradford, M.A., Wall, D.H., Fierer, N., 2014. Biogeographic patterns in below-ground diversity in New York City's Central Park are similar to those observed globally. Proceedings. Biological Sciences 281, 20141988.

Rognes, T., Flouri, T., Nichols, B., Quince, C., Mahé, F., 2016. VSEARCH: a versatile open source tool for metagenomics. PeerJ 4, e2584.

Röttjers, L., Faust, K., 2018. From hairballs to hypotheses-biological insights from microbial networks. FEMS Microbiology Reviews 42, 761-780.

Rousk, J., Bååth, E., Brookes, P.C., Lauber, C.L., Lozupone, C., Caporaso, J.G., Knight, R., Fierer, N., 2010. Soil bacterial and fungal communities across a $\mathrm{pH}$ gradient in an arable soil. ISME Journal 4, 1340-1351.

Saito, R., Smoot, M.E., Ono, K., Ruscheinski, J., Wang, P.L., Lotia, S., Pico, A.R., Bader, G.D., Ideker, T., 2012. A travel guide to Cytoscape plugins. Nature Methods 9, 1069-1076.

Shannon, P., Markiel, A., Ozier, O., Baliga, N.S., Wang, J.T., Ramage, D., Amin, N., Schwikowski, B., Ideker, T., 2003. Cytoscape: a software environment for integrated models of biomolecular interaction networks. Genome Research 13, 2498-2504.

Shen, C., Ni, Y., Liang, W., Wang, J., Chu, H., 2015. Distinct soil bacterial communities along a small-scale elevational gradient in alpine tundra. Frontiers in Microbiology 6, 582.

Shen, C., Shi, Y., Fan, K., He, J.S., Adams, J.M., Ge, Y., Chu, H., 2019. Soil pH dominates elevational diversity pattern for bacteria in high elevation alkaline soils on the Tibetan plateau. FEMS Microbiology Ecology 95, fiz003.

Shen, C., Xiong, J., Zhang, H., Feng, Y., Lin, X., Li, X., Liang, W., Chu, $\mathrm{H}$., 2013. Soil $\mathrm{pH}$ drives the spatial distribution of bacterial communities along elevation on Changbai Mountain. Soil Biology \& Biochemistry 57, 204-211.

Singh, D., Lee-Cruz, L., Kim, W.S., Kerfahi, D., Chun, J.H., Adams, J. M., 2014. Strong elevational trends in soil bacterial community composition on Mt. Halla, South Korea. Soil Biology \& Biochemistry $68,140-149$.

Song, F.Q., Tian, X.J., Li, Z.Q., Yang, C.L., Chen, B., Hao, J.J., Zhu, J., 2004. Diversity of filamentous fungi in organic layers of two forests in Zijin Mountain. Journal of Forestry Research 15, 273-279.

Toju, H., Tanabe, A.S., Sato, H., 2018. Network hubs in rootassociated fungal metacommunities. Microbiome 6, 116.

Trivedi, P., Leach, J.E., Tringe, S.G., Sa, T., Singh, B.K., 2020. Plantmicrobiome interactions: from community assembly to plant health. Nature Reviews. Microbiology 18, 607-621.

Wang, J.J., Soininen, J., 2017. Thermal barriers constrain microbial elevational range size via climate variability. Environmental Microbiology 19, 3283-3296.

Wang, Y., Li, C., Shen, Z., Rui, J., Jin, D., Li, J., Li, X., 2019. Community assemblage of free-living diazotrophs along the elevational gradient of Mount Gongga. Soil Ecology Letters. 1, 136-146.

Wang, Z., Li, M., Zhang, X., Song, L., 2020. Modeling the scenic beauty of autumnal tree color at the landscape scale: A case study of Purple Mountain, Nanjing, China. Urban Forestry \& Urban Greening 47, 126526.

Weiss, S., Van Treuren, W., Lozupone, C., Faust, K., Friedman, J., Deng, Y., Xia, L.C., Xu, Z.Z., Ursell, L., Alm, E.J., Birmingham, A., Cram, J.A., Fuhrman, J.A., Raes, J., Sun, F., Zhou, J., Knight, R., 2016. Correlation detection strategies in microbial data sets vary widely in sensitivity and precision. ISME Journal 10, 1669-1681.

Xu, H.J., Li, S., Su, J.Q., Nie, S.A., Gibson, V., Li, H., Zhu, Y.G., 2014. Does urbanization shape bacterial community composition in urban park soils? A case study in 16 representative Chinese cities based on the pyrosequencing method. FEMS Microbiology Ecology 87, 182-192.

Xu, M., Li, X., Cai, X., Gai, J., Li, X., Christie, P., Zhang, J., 2014. Soil microbial community structure and activity along a montane elevational gradient on the Tibetan Plateau. European Journal of Soil Biology 64, 6-14.

Yang, T., Shi, Y., Zhu, J., Zhao, C., Wang, J., Liu, Z., Fu, X., Liu, X., Yan, J., Yuan, M., Chu, H., 2021. The spatial variation of soil bacterial community assembly processes affects the accuracy of source tracking in ten major Chinese cities. Science China. Life Sciences 64, 1546-1559.

Yuan, Z.S., Liu, F., Zhang, G.F., 2015. Bacterial diversity and community structure in moso bamboo forest soils based on 454 pyrosequencing. Dokladi na Bulgarskata Akademiâ na Naukite 68, 609-619.

Zhalnina, K., Dias, R., de Quadros, P.D., Davis, R.A., Camargo, F A O., Clark, I.M., McGrath, S.P., Hirsch, P.R., Triplett, E.W., 2015. Soil $\mathrm{pH}$ determines microbial diversity and composition in the park 
grass experiment. Microbial Ecology 69, 395 406.

Zhang, B., Zhang, J., Liu, Y., Shi, P., Wei, G., 2018. Co-occurrence patterns of soybean rhizosphere microbiome at a continental scale. Soil Biology \& Biochemistry 118, 178-186.
Zhang, Z., Geng, J., Tang, X., Fan, H., Xu, J., Wen, X., Ma, Z., Shi, P., 2014. Spatial heterogeneity and co-occurrence patterns of human mucosal-associated intestinal microbiota. ISME Journal 8, 818893. 